\title{
4. Nationaler Grippeimpftag am Freitag, 9. November 2007*
}

Eine Initiative der Schweizer Hausärztinnen und Hausärzte: KHM in Zusammenarbeit mit SGAM, SGIM, SGP und FMP, unterstützt und finanziert vom BAG und ideell von der FMH.
Die Schweizer Hausärzte bieten all jenen Risikopersonen, die sich bis anhin noch nicht gegen Grippe impfen liessen, einen leichten Zugang zur Grippeimpfung (präventivmedizinisches Angebot) ohne Anmeldung und mit minimalen Formalitäten zu einem Richtpreis von Fr. 25.-.

Weil die Impfinteressenten in ihrer Umgebung eine Hausarztpraxis suchen, die am Grippeimpftag mitmacht, ist es wünschenswert, dass die Namen der teilnehmenden Praxen und Ärztinnen aktiv bekanntgemacht werden, sei es in den regionalen Medien, an öffentlichen Anschlagbrettern oder im Internet. Wir bitten Sie, liebe Kolleginnen und Kollegen, sich an dieser Aktion zu beteiligen.

Als Idee - damit Impfinteressierte aus den Risikogruppen sichtbar und einfach Ihre Praxis als mitmachende Praxis erkennen - schlagen wir vor, das Grippeimpfplakat vom 9. November 2007 an Ihrem Praxisschild anzubringen.

Auf der Homepage www.kollegium.ch unter der Rubrik «Nationaler Grippeimpftag $\rightarrow$ Unterlagen für Ärzte» finden Sie diverse Mustertexte für die Ankündigung in der Praxis, für Presseeinsendungen sowie Kleinplakate, die Sie für zusätzliche Informationen an die Impfinteressenten benützen können.
Die Durchführung dieses 4. Nationalen Grippeimpftages werden wir nochmals kurz vor dem Grippeimpftag durch Medienmitteilung in Tagespresse, Radio und Fernsehen publik machen.

\section{Kontaktstellen und Adressen für mehr Infos}

\section{Kollegium für Hausarztmedizin KHM}

www.kollegium.ch

Ursula Laubscher, Koordinatorin Grippeimpftag, Geschäftsstelle KHM, Landhausweg 26, 3007 Bern, Tel. 03137006 70/71/72, Fax 031370 06 79, E-Mail: ursula.laubscher@kollegium.ch

\section{Bundesamt für Gesundheit}

www.bag.admin.ch/influenza

Für Bestellungen von Informationsmaterial: Dokumentationsstelle Grippeprävention, Postfach, 3000 Bern 6, Tel. 03135214 60, Fax 03135214 71, E-Mail: grippe@bag.admin.ch, Internet: www.bag.admin.ch $\rightarrow$ Dienstleistungen $\rightarrow$ Krankheiten und Medizin $\rightarrow$ Grippe 